\title{
AN EXPERIMENTAL INVESTIGATION OF DRIFT PROFILES IN A CLOSED CHANNEL
}

\author{
R. C. H. Russell, Principal Scientific Officer \\ and J. D. C. Osorio, Scientific Officer \\ Hydraulics Research Station, Wallingford, England.
}

\section{INTRODUCTION}

The first order theory of water waves states that water particles move in closed orbits; that this was not exactly so, and that there was a slow drift of the water in the direction of wave propagation, was first realised by Stokes $(1)$ who proposed a solution of the horizontal drift or mass transport for waves in an inviscid fluid. Recently the effect of the viscosity has been investigated by (Lpnguet-Higgins (2) Experimenta] measurements have been made by Caligny (3) the U.S. Beach Erosion Board $(4)$ Bagnold (5) and previously by the Hydraulics Research Station. The evidence from these sources is incomplete.

In the experiments described in this paper measurements of the mass transport at all levels were made with progressive waves, varying in length from about $1 \frac{1}{2}$ to $50 \mathrm{ft}$ and in water between 6 and $20 \mathrm{in}$. deep.

\section{SUMMARY OF RESULTS}

It was found that the drift near a horizontal bed was invariably in the direction of wave propagation and quantitatively in good agreement with that predicted by Longuet-Higgins. The drift at the surface was in the direction of wave propagation in every experiment but one, and the return flow took place near the centre of the fluid. In deep water the theory of Stokes predicted the drift velocities. When the ratio of depth to wavelength lay within a certain range defined by $.7<\mathrm{kd}<1.5$ the drift velocities at all depths were in agreement with those calculated from LonguetHiggins "conduction solution" which however is not strictly applicable in the present observations.

It was found that the drift on the seaward and shoreward faces of a submarine bar, that had both faces at slopes of 1 in 20 was insignificantly different from the drift on a horizontal bed; except when the waves were spilling over the bar. When the waves were spilling, the direction of the drift at the bed on the inshore face was reversed: the drift was then towards the crest of the bar from both sides.

\section{Table of Symbols}

$\mathrm{H}$ wave height

a wave amplitude $\mathrm{H} / 2$

a still water depth

$\lambda$ wave length

k $2 \pi / \lambda$

T wave period

$\sigma \quad 2 \pi / \mathfrak{T}$ 


\section{COASTAL ENGINEERING}

$v$ kinematic viscosity

$y$ distance from the surface, measured downwards from still water leve

$\mu \quad y / d$

$U$ drift velocity, taken as positive when the motion is in the directi of wave propagation. Subscripts indicate the value of $\mu$ where the velocity is measured, e.g. $U_{0}$ is the drift velocity at the surface.

Stokes' theory assumes irrotational waves of small but finite heigl propagated in a perfect inviscid fluid. It gives a drift velocity in deep water of

$$
\begin{aligned}
U & =a^{2}\left(\frac{2 \pi}{\lambda}\right)^{3 / 2} g^{\frac{1}{2}} e^{-4 \pi y / \lambda} \\
& =a^{2} \sigma \mathrm{k} \mathrm{e}^{-4 \pi y / \lambda}
\end{aligned}
$$

thus $U_{0}=a^{2} \sigma \mathrm{k}$

All drift velocities are positive; and the condition of no net flon across a section, which is assumed to prevail in a narrow channel, is no satisfied. However, if the axis is shifted arbitrarily, the condition $c$ be satisfied, and this alters the surface drift by only a small proporti if the water is deep. Stokes performed this operation and showed that when $d>\lambda$ the drift velocity at any depth was

$$
U=a^{2} \sigma k e^{-4 \pi u \lambda}-\frac{a^{2} \sigma}{2 d}
$$

hence $U_{0}=a^{2} \sigma k\left(1-\frac{1}{2 k d}\right)$

Stokes also obtained the drift in water of finite depth, but in view of shear at the bed in shallow water this would not be expected to apply.

Ionguet-Higgins' theory applies to wave propagated in a viscous fluid of finite depth, the motion being assumed to be rotational but non. turbulent. The theory predicts a velocity at the bed of

$$
U=\frac{5}{4} \frac{a^{2} \sigma k}{\sinh ^{2} k d}
$$

In this formula there is no restriction on the wave amplitude, provided the flow is laminar. However in an appendix to the present paper he has indicated reasons why the above formula may be applicable also to the case of turbulent flow.

According to Longuet-Higgins, the flow in the interior of the fluid may be expected to depend on the ratio of the rave amplitude to the thickness of the boundary-layer $\delta$, which is of the order of $0.5 \mathrm{~mm}$. When the wave amplitude is small compared with $\delta$ (a condition very rarely satisfied) the drift velocities are given by the "conduction solution" 


\section{IN A CLOSED CHANNEL}

$$
\begin{aligned}
U=\frac{a^{2} \sigma k}{4 \sinh ^{2} k d}\left\lfloor 2 \cosh (2 k d(\mu-1))+3+k d \sinh 2 k d\left(3 \mu^{2}-4 \mu+1\right)\right. \\
\left.+3\left(\frac{\sinh 2 k d}{2 k d}+\frac{3}{2}\right)\left(\mu^{2}-1\right)\right\rfloor
\end{aligned}
$$

But when the wave amplitude is large compared with $\delta$, as was the case in the present experiments, the velocity profile in the interior is not predicted, but is said to depend on the conditions at the boundaries, in this case the wave-generator and the beach.

The physical reason for this as given by Longuet-Higgins is that under normal conditions ( $a \gg \delta$ ) the transport of vorticity by convection along, the streamlines will be much greater than the diffusion of vorticity by viscous conduction. Initially, however, that is to say before any vorticity has had time to penetrate the interior of the fluid, the motion there should be as given by Stokes' irrotational solution.

Both in Stokes' solution and in Longuet-lliggins' conduction solution $a^{2} \sigma k$, which is a velocity, gives the scale of the velocity while the remainder of the expressions show how the velocity varies with depth.

It will be seen from the experimental results presented below that many of the drift profiles happen to agree with those predicted by the conduction solution, although the waves are far too high for it to apply. Similarly other results agree quite well with the Stokes' solution and these are shown superimposed upon it. Neither solution is applicable, the Stokes' solution because it considers only an inviscid flund and the conduction solution because it considers waves of such low height; but both have nevertheless been used where they tend to agree with the experimental results.

\section{SURAARY OF PREVIOUS EXPERIMIENTAL FORK}

In the experiments made by Caligny in 1878, the drift velocities at the bed are in agreement with Longuet-Higgins' theory. In the interior, the velocities are forwards near the bed and the surface and backwards at intermediate levels.

In some experiments made by the U.S. Beach Erosion Board drift velocities were observed under conditions that represented a wide range of $\mathrm{kd}$. When kd > 3 fairly good agreement was found with Stokes' theory. The scatter of points with shallow water waves was so large as to render the results valueless.

Bagnold observed the form of the mass transport curve, and measured drift velocities at the bed. His results differ from those predicted by the Longuet-Higgins theory by a maximum of $15 \%$ and the curves are of the same form as the conduction solution. However only long waves of low amplitude were used. 


\section{COASTAL ENGINEERING}

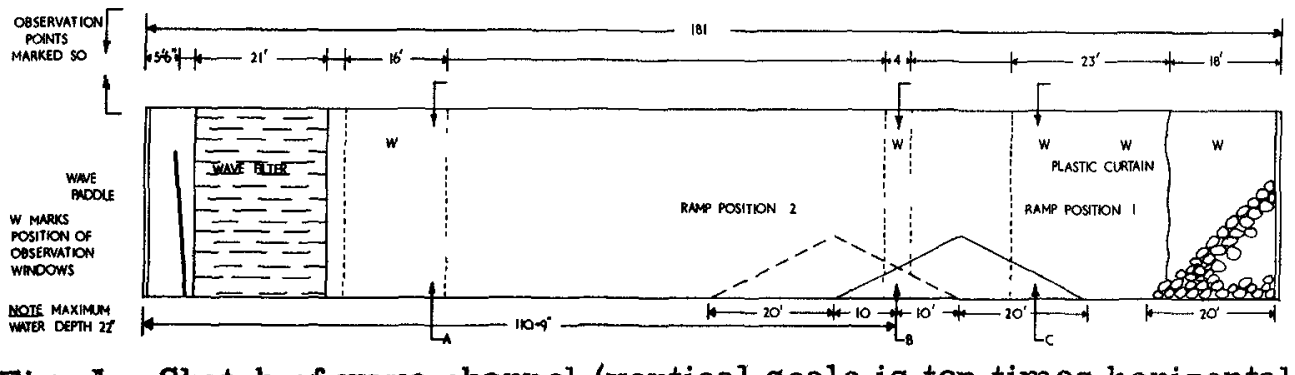

Fig. I. Sketch of wave channel (vertical scale is ten times horizontal scale).

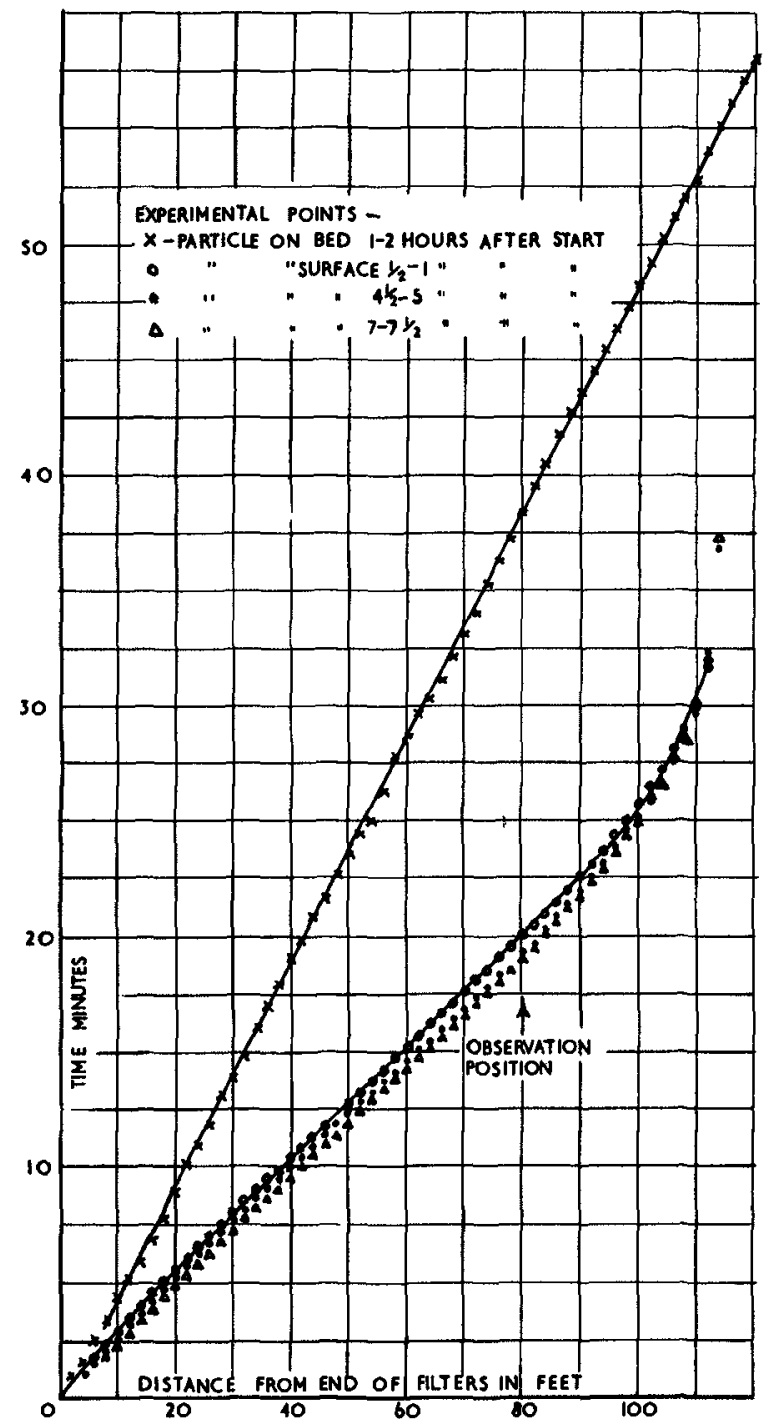

Fig. 2. The variation of drift velocity at the surface and near the bed with distance along the channel $(\mathrm{H}=46$ in $., \mathrm{T}=1.5 \mathrm{sec}, \mathrm{d}=20 \mathrm{in}$.$) .$ 


\section{AN EXPERIMENTAL INVESTIGATION OF DRIFT PROFILES}

\section{IN A CLOSED CHANNEL}

The Hydraulics Research Station carrzed out some earlier experiments in a 54-ft long wave channel, in which drift velocities were obtained by observing the motion of compounded particles having the density of water. A series of curves, mostly " $\mathrm{S}$ " shaped, were obtained with a forward drift at the bed and a backward drift at the surface. The present series of experiments indicate that the channel was too short; and that the results were affected by circulations set up by the waves when they broke.

\section{ATPARATUS}

The wave channel is $185 \mathrm{ft}$ long $4 \mathrm{ft}$ wide and will contain water having a maximum depth of 22 in. Windows are provided at intervals along one side of the channel. The walls are vertical, plane and parallel and the bed is horizontal to whthun limits of $1 / 20$ in. At the wave-generator end of the channel the width increases to $6 \mathrm{ft}$ and the $21-\mathrm{ft}$ long tapered part of the channel is fitted with filters made of curved perforated plates.

The wave fenerator is a paddle, driven by a two-speed induction motor through an infinitely variable friction gear-box and a quick-return mechanism. The linkage allows the paddle movenent to simulate that of a paddle hinged at the bed or at an infinite depth below the bed, or at intermediate levels.

At the other end of the channel is a beach of $\frac{\pi}{6}$-in. shingle with 3-in. shingle on the surface. It has a slope of 1 in 10.

A twin-wire reslstance wave recorder that is sensitive to changes in water level of $1 / 5000 \mathrm{ft}$ is set in a hollow at the back of the shingle beach. At this point the fluctuations of water level caused by the generated waves are small but seiches in the channel arrive with little attenuation.

For taking photographs of the water motions a Rolleiflex camera is used, the shutter operated by a solenoid. The solenoid is actuated by a small pendulum that is moved to and fro by the waves some distance along the channel, and ensures that all photographs of the same waves are taken at precisely the same phase.

\section{METHODS OF MEASURTMENT}

In these experiments the principal method of recording drift velocities involved the timing of small particles that had the same density as water as they travelled known distances along the channel. The particles were normally followed for a period of between 20 and 40 secs but exceptionally the period was as short as 15 secs or as long as 80 secs. The maximum error in the measurement of this period was of the order of 1 wave period. The mean depth of the particle was estimated from horizontal grid lines drawn both on the glass and on the other wall of the channel, and could involve a maximum error of $\frac{1}{4}$ in. Particles which rose or fell more than 1 in. during, an observation were ignored. The lamps illuminating the particles were shaded so that only particles near the centre of the channel were apparent. The illuminated slice of water was 6 in. wide at the surface increasing to $1 \mathrm{ft}$ at the bed. The particles were scattered into the 


\section{COASTAL ENGINEERING}

water near the observation point before an experiment began. They beca diffused throughout the fluid and it was only necessary to wait for a particle to appear at any desired level. Drift profiles were plotted $f$ at least 25 velocity measurements of individual particles.

A second method of recording drift velocities was used when there a seiche in the channel. The seiche had a period of 51 secs and obscur the drift velocities, when they were found by observing particles. The method involved photographing a dye streak as it was deformed by the ar The method had the advantage that a selche might carry the whole dye streak laterally; but the true drifts could still be outalned, if it wa assumed that the drift profile had to be a balanced curve involving no flow in either direction. (The velocities at the very bottom, which ar theoretically not influenced by the seiche, were not treated in this wa: The dye streak was of fluoresce in and was obtained by coating a neutral. buoyancy particle with fluorescein powder, damping it slightly and arop. ping it into the water. A fine bright streak was obtained.

In order to disperse the film of dirt which invariably formed on th surface of the water, Teepol was added until the surface drift velocitit became stable and uniform across the section. Experience soon showed tr amount required.

\section{DILITITIARY EXPERTMINTS}

These were experiments desicned to ensure that the drift velocities obtained were not affected by proximity to the wave generator, to the beach or to the sides of the channel. Observations were also made on th stability of waves as they travelled down the channel, on seiches that $w$ sometimes set up, on the degree of turbulence in the water and on the ti taken to achieve steady conditions.

End Effects. Wach time the wave length was altered by a large amou a float was tined as it drifted on the surface from the filters down the channel. Fig. 2 shows the results of one such experiment. For wave periods between $1 \frac{1}{4}$ and 3 secs it was found that the waves breaking on th beach caused a very strong backward drift at the surface, which apprecia distorted the drift profiles as far back as the observation window $B$. A the paddle end of the channel the uniform drift persisted up to $10 \mathrm{ft}$ fr the filters. Actually at the filters there was no observable drift. Th strong drift initiated near the beach was greatly reduced by installing near the beach a flexible plastic curtain in the waves, which hung from floating wooden bar at its upper edge and carried an iron bar at its low edge. The device was moored by feeble elastic threads. The device had no apparent effect on the waves but ensured that there was zero drift at the point where it was installed. The experiment to which Fig. 2 refers was made with olastic curtain in place. The figure shows that the surfa drift is uniform over a distance of $80 \mathrm{ft}$ and that the observation windor is suitably placed for measuring it. The uniformity of the surface drif. along the greater part of the channel with divergences only at the ends was taken by us to indicate that the drift was not in fact determined by conditions at the ends of the channel but were independent of them. Fig. contains five drift profiles all for the same wave conditions and depth 


\section{IN A CLOSED CHANNEL}

of water, but measured at different places in the channel. It is clear that only close to the beach or very close to the glass window is the drift profile significantly altered.

Side Effects. Floats were dropped Into the water simultaneously on a line parallel to the wave crests and it was observed that except for the floats within 2 in, from the walls they remained in a straight line. Those close to the walls noved ahead faster than the others. It can be seen that floats move away from the well when the water 1 s rising and towards the wall when the water is falling: they are furthest from the wall when the water is moving forward and closest to the wall when the water is movino backwards. This is sufficient to explain their additional drift velocity. The movement towards and away from the wall is thought to be caused by the phase lead of the motion near the wall on the motion elsewhere $(6)$ and is associated with small vortices having their axes parallel to the axes of the channel at the intersection of the water surface with the wall.

Steady Conditions. Experinents were made to determine the time required to achieve steady conditions. If stability was to be achieved, the time varied with the waves that were produced but seldom exceeded one hour and never exceeded three hours. There was one series of experiments in which the drift never settled down, one of the experiments being continued for seven hours without the drift showing any signs of becoming steady. In these experiments which related to very long waves, kd was 0.29 , the drift velocity at the bed was steady and was recorded.

Fig. 2 relates to the more normal experiments where steady conditions were achieved in the first hour. The figure shows by the parallelism of the lines at the observation point that the surface drift there was the same after $I$ hour as after 5 hours and $7 \frac{1}{2}$ hours.

Stability of the Waves. It was found that waves with perlods shorter than $\frac{5}{4}$ sec were unstable and about half-way along the channel broke up into groups of five waves with almost undisturbed regions, a wavelength long, between them. The effect appeared with longer waves if the height was increased. That the effect was not caused by irregularities in the speed of the wave generator was verified with a stroboscope. Drift profiles with these short waves were observed at point $A$ shown on Fig. 1 , where the waves were still of constant height.

Seiches. When the wave perlod exceeded 3 secs it was found that the seiche that was set up when the wave-generator was started did not die down after $20 \mathrm{~min}$, as was otherwise the case, but reached a steady height between .001 and $.003 \mathrm{ft}$. The seiches resulted in a variation of velocity of up to $I_{\frac{1}{2}} \mathrm{ft}$ per minute during their 51 secs period and made it impossible to obtain systemmatic results from the observation of particles. The photographic method was therefore used and the results were adjusted where necessary for no net flow across the section.

Turbulence. Dye streaks were observed under all conditions in order to obtain the general form of the curves: most of them were photographed. The dye streaks remained intact and undiffused for very long periods - 


\section{COASTAL ENGINEERING}

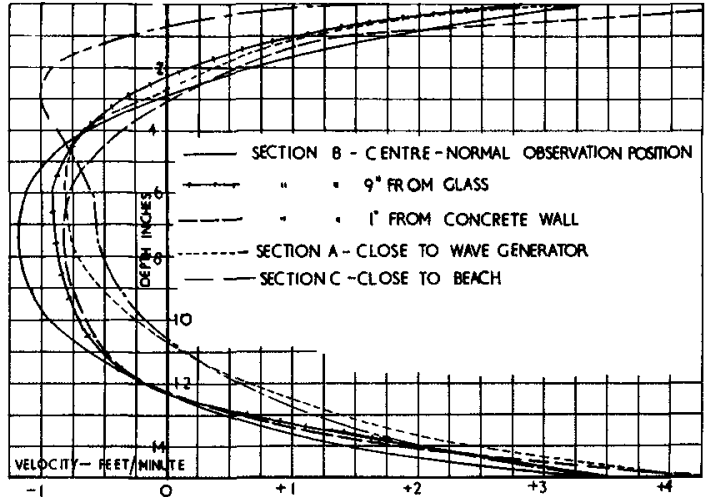

Fig. 3

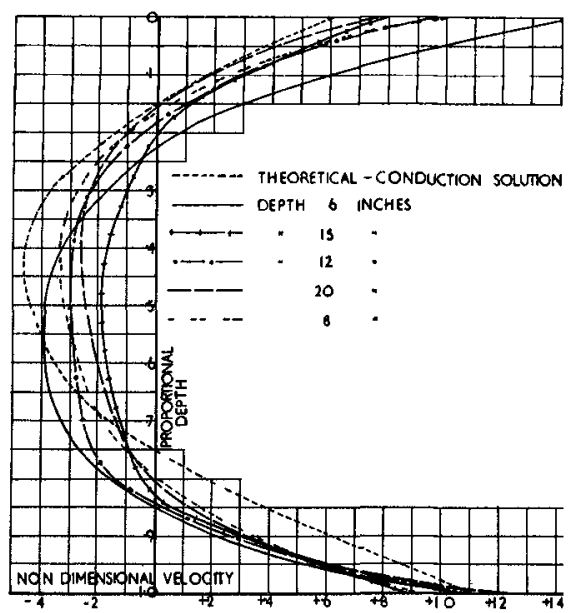

Fig. 4

Fig. 3. Drift profiles at different places in the channel $(H=3.6$ in., $\mathrm{T}=1.5 \mathrm{sec}, \mathrm{d}=15 \mathrm{in}$. ).

Fig. 4. The variation of non-dimensional drift profile with scale $(a / d=$ $0.23, \mathrm{kd}=0.92$ ).

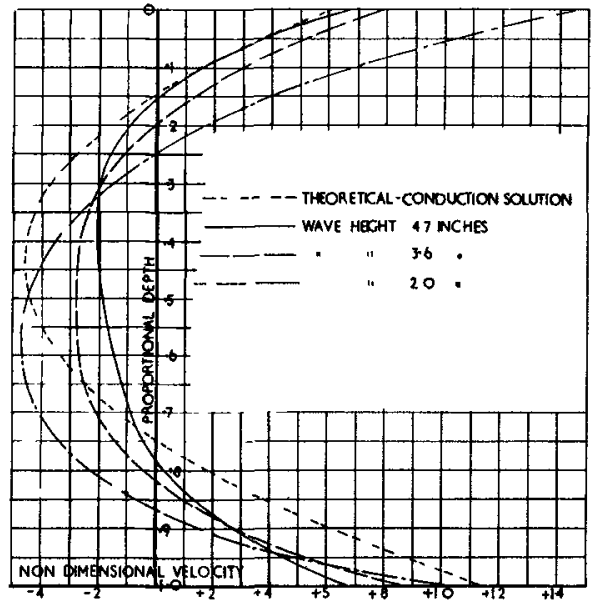

Fig. 5

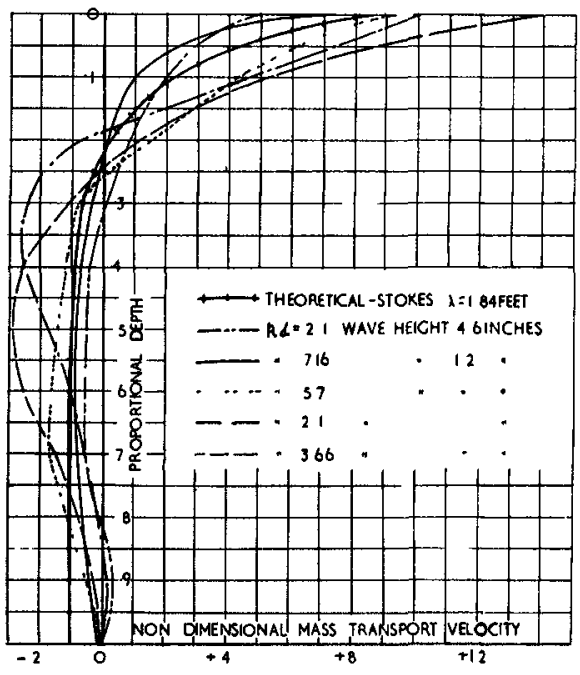

Fig. 6

Fig. 5. The variation of non-dimensional drift profile with wave-heigh $\mathrm{T}=1.5 \mathrm{secs}, \mathrm{d}=15 \mathrm{in}$. ).

Fig. 6. The variation of non-dimensional drift profile with $\mathrm{kd}$. Deep water waves ( $d=20$ in .). 


\section{IN A CLOSED CHANNEL}

indicating that the flow was laminar - except within 1 in. of the bed, where a diffuse cloud of dye was formed whenever $\mathrm{kd}<1.5$. The turbulent layer was thicker for higher velocities at the bed. It should be noted that in the turbulent layer near the bed the dye spreads both forwards and backwards; and that the advance of the front of the cloud is faster than the drift indicated by a particle.

\section{EXFL ?TIENTAL RESULIS}

Fig. 4 shows the results of a series of experiments using geometrically similar waves, designed to show whether the drift profiles scaled according to the ratio $a^{2} \sigma_{k}$. Each experiment was done with a different depth of water but the ratios $a / d$ and $\lambda / d$ were kept constanto The drift velocities should be proportional to $\mathrm{a}^{2} \sigma \mathrm{k}$ : accordingly if the measured drift velocities are divided by $a^{2} \sigma k$, and plotted against $y$, the proportional depth, ldentical curves should result. The figure shows that except when the depth of water was as little as 6 in. the profiles were similar and close to the profile of Longuet-Higgins' conduction solution.

An experiment with a water depth of only $4 \mathrm{in}$. foiled to produce a repeatable curve. Although a dye streak indicated a proflle similar to those in Fig. 4, there was a greater forward drift on the surface down one side of the channel than down the other; indicating a large scale circulation in the horizontal plane. It is possible that this will always be the case when the width of the channel is large compared with the depth of water.

Fig. 5 shows the results of experiments to determine whether drift velocities were strictly proportional to $\mathrm{a}^{2}$. It is found that when plotted non-dimensionally the low waves result in faster drifts than the high waves; and this indicates that the velocities are proportional to rather less than the second power of a.

Fig. 6 contains drift profiles for deep water waves in a depth of 20 in., measured at Section $A$ in the channel. The agreement with Stokes' theory, modified to produce a curve balanced about the axis, is good except near the bed. The conduction solution for these conditions gives surface velocities many times too big. It was thourht possible that a different drift profile might be obtained if an experiment were run continually for the time $d^{2} / v$ that would be needed for the conduction solution to apply. To test this hypothesis an experiment was run for 24 hrs with water $11 \frac{1}{2}$ in. deep, so that $\mathrm{d}^{2} / \nu$ was $18 \mathrm{hrs}$. It was found that the surface drift had a slight tendency to decrease after 6 hrs running, but remained close to the Stokes value.

Figs. 7 and 8 show the observed drift velocities for $k d$ values of 0.5 and 1.25. The scatter of points can be seen. The conduction solution is shown for comparıson. The experiment to which Fig. 7 refers was the only one in which a negative drift at the surface was found.

Fig. 9 is similar to Fig. 6 but relates to shallow water. It shows the variations in the drift profiles for different values of $k d$. They are in qualitative agreement with Longuet-Higgins' conduction solution where 


\section{COASTAL ENGINEERING}

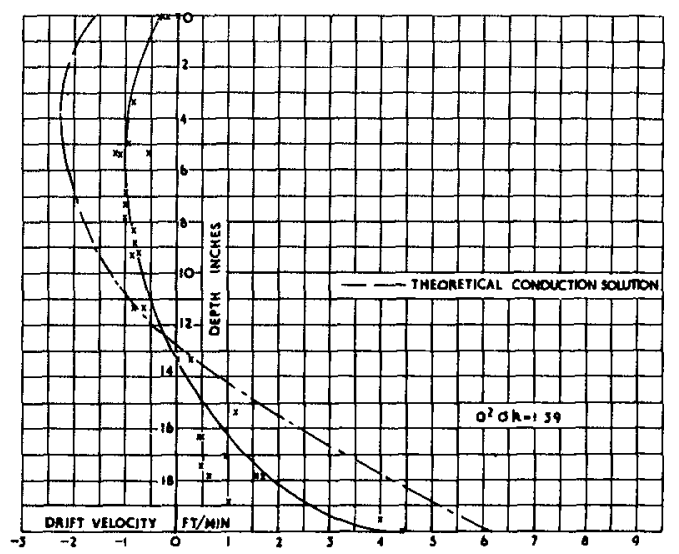

Fig. 7

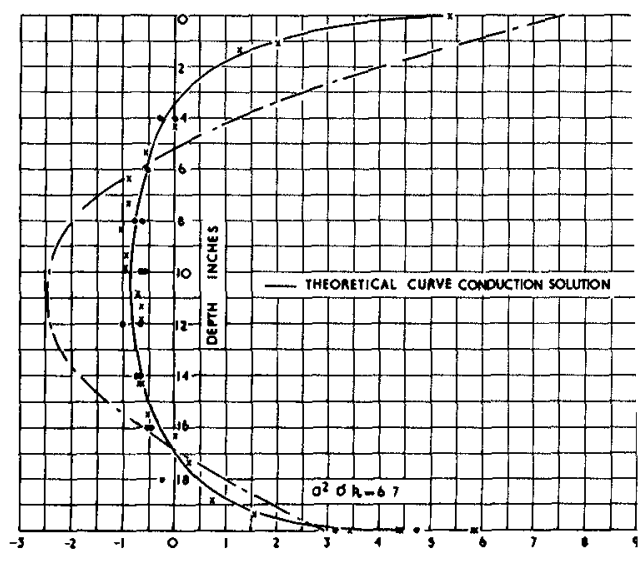

Fig. 8

Fig. 7. Comparison between the experimental and theoretical drift pr file when $\mathrm{kd}=0.5(\mathrm{H}=4.6 \mathrm{in} ., \mathrm{T}=3 \mathrm{secs}, \mathrm{d}=20 \mathrm{in}$.$) .$

Fig. 8. Comparison between the experimental and theoretical drift pr file when $\mathrm{kd}=1.25$ ( $\mathrm{H}=4.6 \mathrm{in} ., \mathrm{T}=1.5 \mathrm{secs}, \mathrm{d}=20$ in .).

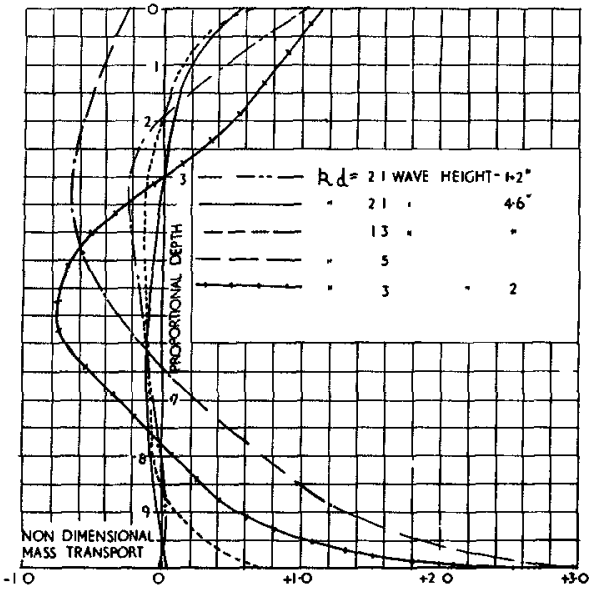

Fig. 9

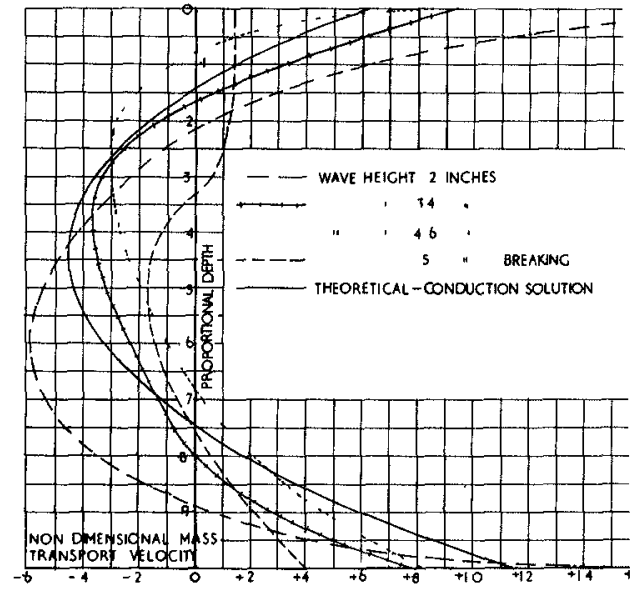

Fig. 10

Fig. 9. The variation of non-dimensional drift profile with kd. Shallov water waves $(d=20$ in.).

Fig. 10. The variation of non-dimensional drift profile with wave-heig] the bed having a positive slope of 1 in $20(T=1.5 \mathrm{secs}, d=1 \mathrm{f}$ $\mathrm{kd}=0.92$ ). 
AN EXPERIMENTAL INVESTIGATION OF DRIFT PROFILES

IN A CLOSED CHANNEL

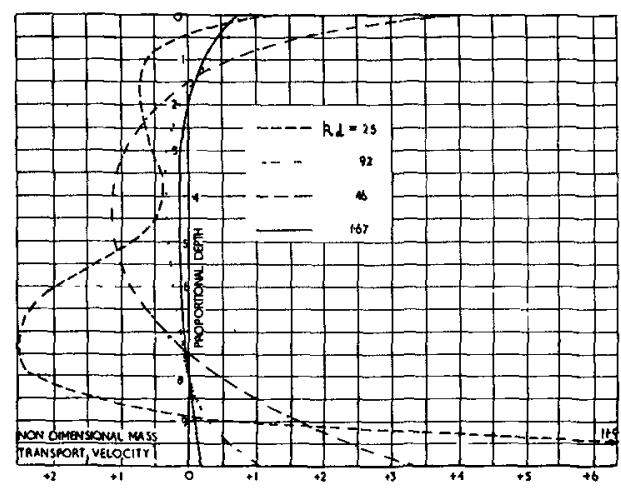

Fig. 11

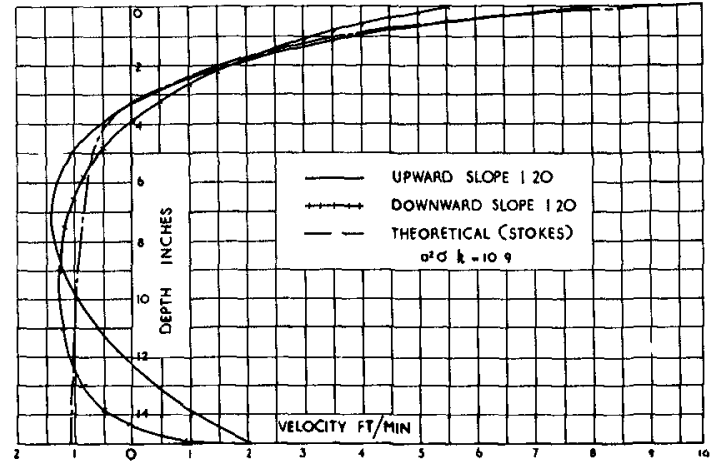

Fig. 12

Fig. 11. The variation of non-dimensional drift profile with kd. Shallow water waves on a positive slope of 1 in $20(\mathrm{H}=3.6 \mathrm{in} . \mathrm{d}=15 \mathrm{in}$.$) .$

Fig. 12. The variation of non-dimensional drift profile with slope $(H=$ 3.4 in., $\mathrm{T}=1 \mathrm{sec}, \mathrm{d}=15 \mathrm{in} ., \mathrm{kd}=1.67)$.

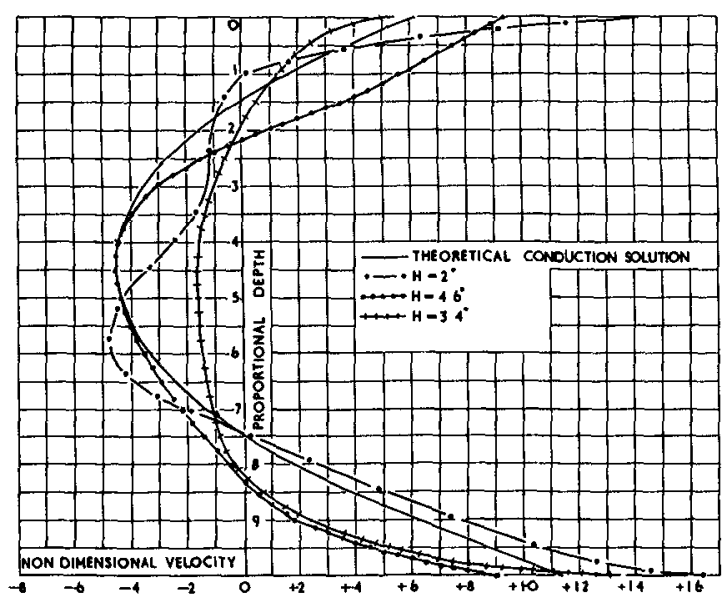

Fig. 13

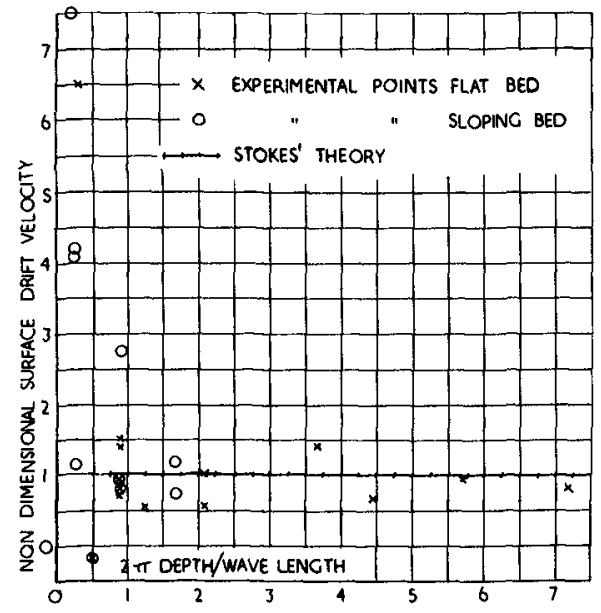

Fig. 14

Fig. 13. The variation of non-dimensional drift profile with wave-height, the bed having a negative slope of 1 in $20(\mathrm{~T}=1.5 \mathrm{sec}, \mathrm{d}=15 \mathrm{in}$., $\mathrm{kd}=0.92$ ).

Fig. 14. Comparison between theoretical and experimental non-dimensional surface drifts. 


\section{COASTAL ENGINEERING}

$k d>0.5$. When $k d<.3$ the profiles varied unsystemmatically with smal: varlations in wave characteristics and usually involved flows in directions other than in the direction of wave propacation. Mlany of the drift profiles became stable in $15 \mathrm{~min}$ : others were unstable and altered continuously. The single curve that had approximately the theoretical form when $k \bar{d}=.3$ was a very low wave, only $2 \mathrm{in.}$ high. Although at the low values of $k d$ the drift in the body of the fluid varied unsystemmatically, that at the bed was consistent and was recorded. The velocities are assembled with others in wig. 15.

F.2. 10 and 11 are similar to 1 L. 5 and 9 except that they relate waves running up a slope into shallower water (positive slope). The def of vrater, $d$, is taken to be that in the midale of the slope where the observations were made. Unless the waves broke on the crest of the bar, the drift profiles were little affected by the slope.

Fig. 12 shows that the effect of a change in slope from $+1 / 20$ to $-1 / 20$ is smell for short waves.

Fig. 13 is similar to Fir. 10 and 5 but relates to a downward slope of 1 in 20. Except for the wave $5 \mathrm{in}$. high that broke on the crest of $t$ bar the profiles are not very different. The 5-in. breakin wave caused a strong forward drift at the surface and a strong negative drift at the bed. There was so much turbulence that no observations on the drift in the body of the fluid could be made. Other experiments performed on the downward slope, with $k$ d <.9, failed to produce stable drift proriles: It was found however that when the waves broke near the crest there was invariably a negative drift on the bed as far back as the point at which the waves broke; and a forvard drift at the surface. If the waves spill only very slishtly the bea drift could be in the positive direction.

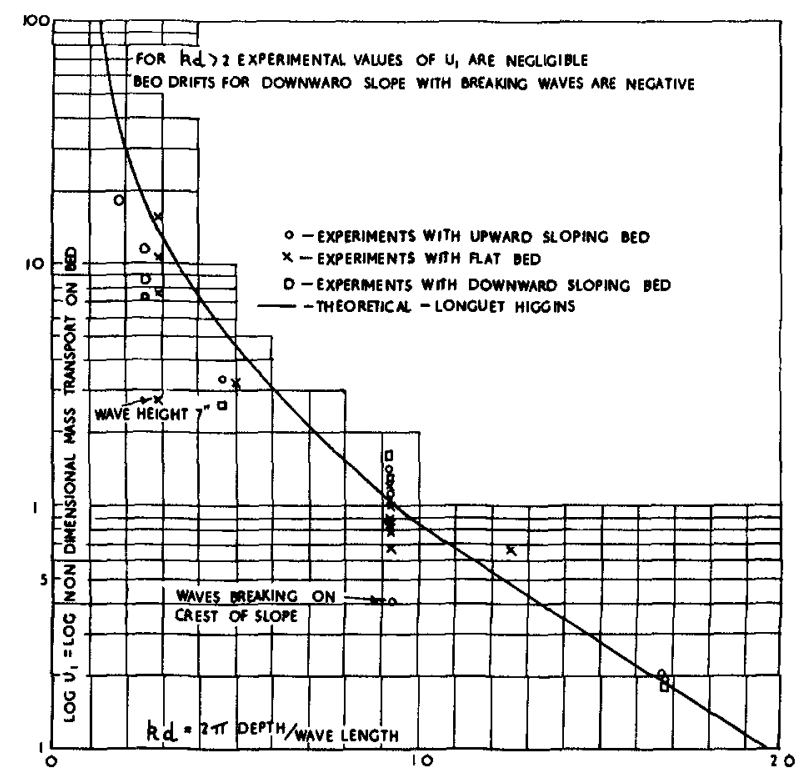

Fig. 15. Comparison between the experimental non-dimensional drift at the bed and that according to Longuet-Higgins . 


\section{AN EXPERIMENTAL INVESTIGATION OF DRIFT PROFILES}

\section{IN A CLOSED CHANNEL}

\section{COHCLUSIOIS}

It was found possible to obtain drift velocities set up by progressive waves in a closed channel, which were independent of nosition and time. It is probable however that the velocities would be disordered by circulations in a horizontal plane, if the waves were not confined to a narrow channel.

Stable drift profiles were not obtained with the very longest waves, those amountine to a succession of solitary waves, except when the waves were very low. Possibly the channel, which was only long enough to contain four of these waves at a tine, was too short.

Near the bottom the drift velocities are as predicted by LonguetHiggins for all vaues of $k d$ that were investigated. Fig. 15 shows this agreement. This is in spite of the fact that the theory applies in the first place to laminar conditions, whereas the flow was nearly always turbulent. However in an appendix Longuet-Higgins has given reasons why the formula may be generally applicable in the turbulent case also.

For the surface and interior of the fluid there is no strictly applicable theory. However in deep water the surface drifts are found to be as in Stokes' irrotational theory. Further, when $0.7<\mathrm{kd}<.13$ the profiles in the interior are fairly well fitted by Longuet-Higgins' conduction solution. There is only one departure from this curve which is systemmatic. It is that for a given value of $k d$ the lower waves produce bigger non-dimensional drift velocities.

The drift profiles are disrupted in the neighbourhood of breaking waves. This provides a mechanism capable of sustaining an off shore sand bar, because opposed bed drifts are set up which meet at the top of the bar.

When the waves do not break, the slope of the bed does not alter the drift curves a great deal and the theoretical values of the drift at the bed are almost equally applicable to waves over a horizontal or a gently sloping bed.

\section{PEFERENCES}

(1) Stokes, G. G. On the theory of Oscillatory Waves. Trans. Cambridge Phil. Soc., Vol VIII, p.44I, read lst Narch 1847 \& Vol. IX, p.20, 1851.

(2) Longuet-Higgins, M.S. Mass Transport in Water Waves. Phil. Trans. Roy. Soc. London, Series A, No. 903, Vol. 245, pp.535-581, 31 st hiarch 1953.

(3) Caligny, A. F. H. de C. R. Acad. Sci., Paris Vol. 87, 10, 1878.

(4) U.S. Beach Erosion Board. A Study of Progressive Oscillatory Waves in Water. Tech. Report No. I, U.S. Govt. Printing Office, 1941.

(5) Bagnold, R. A. Sand hiovement by Waves: Some Small Scale Experiments with Sand of very Low Density. Jour. I.C.E., No. 4, 1946-47, p. 447 .

(6) Lamb, H. Hydrodynamics. 6th Revised Edition. Chap. XI, para 347. 183 


\title{
COASTAL ENGINEERING
}

\section{APPENDIX}

THE MECHANICS OF THE BOUNDARY-LAYER NEA? THE BOTTOM IN A PROCRESSIVE W.

\author{
M. S. Longuet-Higgins \\ National Institute of Oceanography, \\ Wormley, England
}

Mr. Russell has asked me to give a brief theoretical account of th somewhat paradoxical forwards drift in the boundary-layer near the bott which he and Mr. Osorio have measured. A general treatment of such boundary-layer effects is to be found in a previous paper (2); but in th following I shall try to give a simple physical picture of one particul case, namely where the wave motion is purely progressive, and the bottc is rigid and level.

It is assumed at first that the viscosity is constant and that the motion is laminar - a condition not always satisfied in Mr. Kussell's experiments. Under these circumstances it is show that the mass-trans port velocity near the bottom and just outside the boundary-layer is given by

$$
U=\frac{5 A^{2}}{4 c}
$$

where $A$ is the amplitude of the horizontal oscillatory motion at the bottom and $c$ is the wave velocity. Since, however, the observations are in agreement with this result even when the flow is turbulent, I also consider the case where the (constant) coefficient of viscosity is replaced by a coefficient of eddy viscosity depending on the distance from the bottom. I find then that the above formula is valid independently of the functional form of the viscous coefficient. This appears to be a step towards the explanation of the phenomenon in the turbulent case.

(1) The boundary layer at the bottom According to the first-order theory of surface waves, and from observation, a wave in water of finit, depth produces near the bottom a horizontal oscillatory velocity given 1

$$
u_{\infty}=A \cos (\sigma t-k x)
$$

approximately, where

$$
A=\frac{a \sigma}{\sinh k d} \text {. }
$$

However, on the bottom itself the velocity must be zero. It appears, then, that there is a region of strong shear very close to the bottom, where viscous stresses are important, and outside which they are relatively small. This region may be called the "boundary-layer".

To determine the horizontal motion within this layer, compare an element of f'luid within the layer with an element just outside. (Fig. Al) The forces accelerating each element horizontally are the pressure 
AN EXPERIMENTAL INVESTIGATION OF DRIFT PROFILES

IN A CLOSED CHANNEL
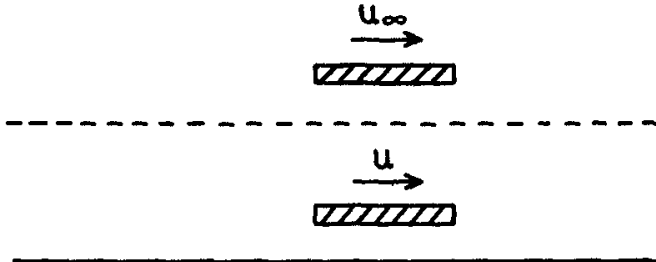

Fig. A1. Comparison of the motion of two fluid elements in and outside the boundary-layer.

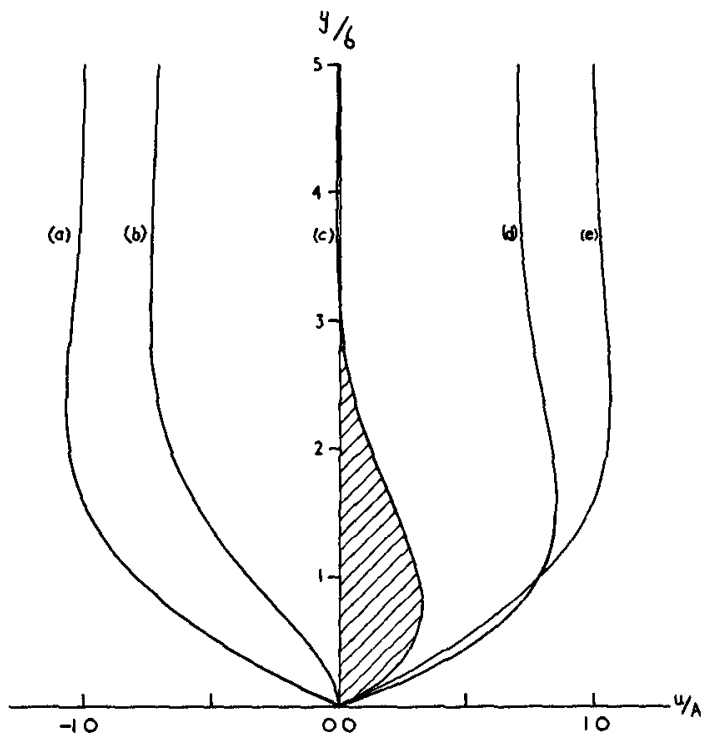

Fig. A2. The velocity-profiles (correct to first order) in the boundarylayer for five successive phases of the motion at intervals of $\mathrm{T} / 8$. (Vertical scale greatly exaggerated).
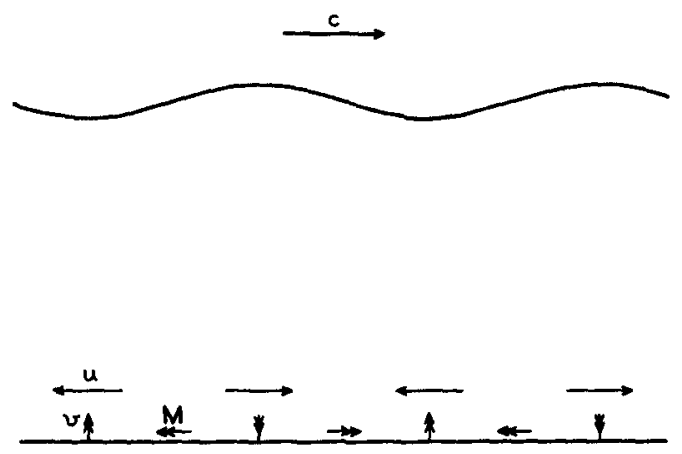

Fig. A3. Diagram showing the origin of the vertical motion in the boundary-layer. 


\section{COASTAL ENGINEERING}

gradient $\partial \beta / \partial x$ and the viscous stress $\partial / \partial y(\rho \nu \partial u / \partial y)$. Now since the layer is very thin and the vertical acceleration is not large, the pres gradient is practically the same for the two elements, while the viscor stress is appreciable only for the element within the layer. So the difference in their horizontal acceleration is due to the viscous stres only. Hence (neglecting second-order terms)

$$
\frac{\partial u}{\partial t}=\frac{\partial u}{\partial t}+\frac{\partial}{\partial y}\left(\nu \frac{\partial u}{\partial y}\right) \text {. }
$$

When the viscosity is constant, the solution of this equation is

$$
u=A\left[\cos (\sigma t-k x)-e^{-y / \delta} \cos (\sigma t-k x-y / \delta)\right]
$$

where

$$
\delta=\left(\frac{2 \nu}{\sigma}\right)^{1 / 2}
$$

(Here $y$ is measured vertically upwards from the bottom).

The motion is illustrated in Fig. A2, where the velocity profile $i$ shown for various phases, at a fixed point. Effectively the velocity $i$ the same as for a uniform fluid oscillating in the neighbourhood of a plane wall (see Lamb(5) 347). The velocity tends very rapidly to its value $u_{\infty}$ just outside the layer, and the total thickness of the layer $i$ of the same order as $\delta$.

An important feature of the motion is that the phase of the veloci. inside the layer tends in general to be in advance of the velocity just outside. The integrated flow in the layer increases indefinitely, of $\mathrm{cr}$ as $y$ tends to infinity. But the component of the integrated flow which is in quadrature with $u_{\infty}$ is finite, and is given by the shaded area of the velocity-profile curve (c) In Fig. A3. Denoting this by $M$ we have

$$
M=\int_{0}^{\infty} u d y=\frac{1}{2} A \delta \sin (\sigma t-k x) \text {. }
$$

Now if the flow were uniform horizontally, as in Lamb's solution just mentioned, there would be no vertical comoonent of motion. But sir $u$ varies sinusoidally with $x$, so also does the total flow $M$; this produces a piling-up of mass within the layer which gives use to a small but important vertical comnonent of velocity. From Fig. A3 we see that just behind a crest the flow $M$ is nepative, and just in front of a crest it is positive. Hence beneath the crest itself there is "stretching" of the layer, giving a downwards velocity. Simlarly beneath a trough ther is a piling-up in the layer, giving an upwards velocity. Analytically, we have

$$
v=\int_{0}^{y} \frac{\partial v}{\partial y} d y=\int_{0}^{y}\left(-\frac{\partial u}{\partial x}\right) d y=-\frac{\partial}{\partial x} \int_{0}^{y} u d y .
$$

Paying attention only to the part of $v$ that is in phase with $u_{\infty}$, that $i$ the part arising from $M$ we have 


$$
\begin{gathered}
\text { IN A CLOSED CHANNEL } \\
v_{\infty}=-\frac{\partial M}{\partial x}=-\frac{1}{2} A k \delta \cos (\sigma t-k x) .
\end{gathered}
$$

This shows that the mean value of the product $u_{\infty} v_{\infty}$ is negative:

$$
(\overline{u v})_{\infty}=-\frac{1}{4} A^{2} k \delta<0 \text {. }
$$

the significance of which will soon become apparent.

(2) The mean stress on the bottom If first-order terms only are considered, the mean stress on the bottom is identically zero. But we shall show, by a straightforward consideration of momentum, that to second order, the mean stress must in fact be positive.

Imagine a rectangle, one wavelength long, drawn in the fluid with its upper sideCD just outside the boundary-layer and its lower side C'D' on the bottom. When fluid having a horizontal velocity $u$ crosses the upper sideCD with velocity $v$ there is a transfer of momentum across the boundary at the rate puvper unit horizontal distance. The mean rate of transfer of momentum across $C D$ in this way is given by $\overline{u v}$, the familiar Reynolds stress. Consider then the momentum balance inside the rectangle $C D D^{\prime} C^{\prime}$. Along the upper side viscous stresses are negligible and there is a transfer of momentum due to the Reynolds stress $p(\overline{u v})_{\infty}$. On the lower side $C^{\prime} D^{\prime}$ the Reynolds stress vanishes (since $v \approx 0$ ), but there is a mean viscous stress $\rho(\nu d u / \partial y)$. On the two vertical sides the conditions are identical by the periodicity of the motion, and so the transfer of momentum across one side just cancels the transfer across the other side. But the total momentum within the rectangle remains unchanged; therefore the viscous stress on the bottom must just balance the Reynolds stress at the top. In other words

$$
\left(\overline{\frac{\partial u}{\partial y}}\right)_{0}=-(\overline{u v})_{\infty} \text {. }
$$

We have seen from eqm. (6) that the mean product ( $\overline{u v})_{\infty}$ is negative. In other words there is a downwards transfer of momentum into the boundarylayer. To balance this, there must be a backwards stress on the layer at the bottom, that is to say a forwards gradient of mean velocity. In the case when the viscosity is constant we have

$$
\frac{\partial \bar{u}}{\partial y}=-\frac{1}{\nu}(\overline{u v})_{\infty}=\frac{A^{2}}{2 c \delta}>0 .
$$

The velocity gradient at other levels within the layer may be obtained by considering a rectangle CDD"C" (see Fig. A4) which has its lower side C"D" at an arbitrary level within the boundary layer. The same considerations of momentum apply, but now account must be taken of both the viscous stress and the Reynolds stress at the level C"D". This leads us at once to the relation

$$
\overrightarrow{\nu \frac{\partial u}{\partial y}}=\overline{u v}-(\overline{u v})_{\infty} .
$$




\section{COASTAL ENGINEERING}

If the viscosity is given, the profile of the mean velocity $\bar{u}$ may be de by direct integration. This 1 s done in $\$ 5$, and we find

$$
\bar{u}=\frac{1}{c} \overline{u_{\infty} u}+\frac{1}{2 c} \overline{u^{2}}-\overline{\frac{\partial u}{\partial y} \int v d t}
$$

a result whych does not depend upon the distribution of viscosity withis the layer. For constant viscosity we obtain the left-hand curve shown in Fig. A5.

(3) The mass-transport velocity It is essential to distinguish betwee the mean velocity $\bar{u}$ measured at a fixed point and the mass-transport velocity $U$, which may be defined as the mean velocity of the same particle of fluid averaged over a complete period (both $\bar{u}$ and $U$ being assumed small compared with the orbital velocity $u$ ). For examole in $t$ Stokes Irrotational wave the mass transport velocity is always positive relative to the mean velocity. This is for two reasons: first because as a wave crest passes overhead the orbital velocity is positive, and so the particle "stays with the wave", spending slightly longer on the forwards part of its orbit than on the backwards part; secondly, the velocity of a particle is slightly greater at the top of its orbit, wher it is travelling forwards, than at the bottom, where is is travelling backwards (see Fig. A6).

The same considerations apply, in general, in the boundary-layer; although the vertical displacements are very small, the vertical gradier of velocity is correspondingly large, so that both the effects just mentioned become appreciable. However, the phase difference between horizontal and vertical components of velocity is a function of the mean position of a particle within the layer.

Analytically, if $P$ is the point on the orbit of a particle whose me position is $Q$, the instantaneous velocity at $P$ will differ from that at $Q$ by an amount

$$
\Delta u=\frac{\partial u}{\partial x} \Delta x+\frac{\partial u}{\partial y} \Delta y
$$

where $\Delta x, \Delta y$ are the horizontal displacements of $P$ from $Q$. These displacements are given by

$$
\Delta x=\int u d t, \quad \Delta y=\int v d t
$$

approximately, (apart from a constant term).

Hence the difference between the mean velocity of the particle and the velocity at $Q$ is given to the second approximation, by

$$
U=\bar{u}+\overline{\frac{\partial u}{\partial x} \int u d t}+\overline{\frac{\partial u}{\partial y} \int v d t} .
$$

On the bottom itself, $u, v, \partial u / \partial x$ and $\partial v / \partial y$ all vanish, and so on differentiation we find

$$
\frac{\partial U}{\partial y}=\frac{\partial \bar{u}}{\partial y}>0
$$



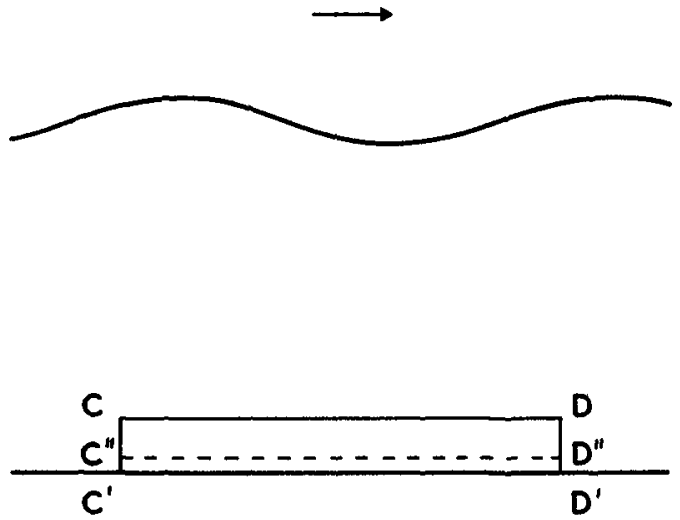

Fig. A4. Diagram for deriving the stress on the bottom.

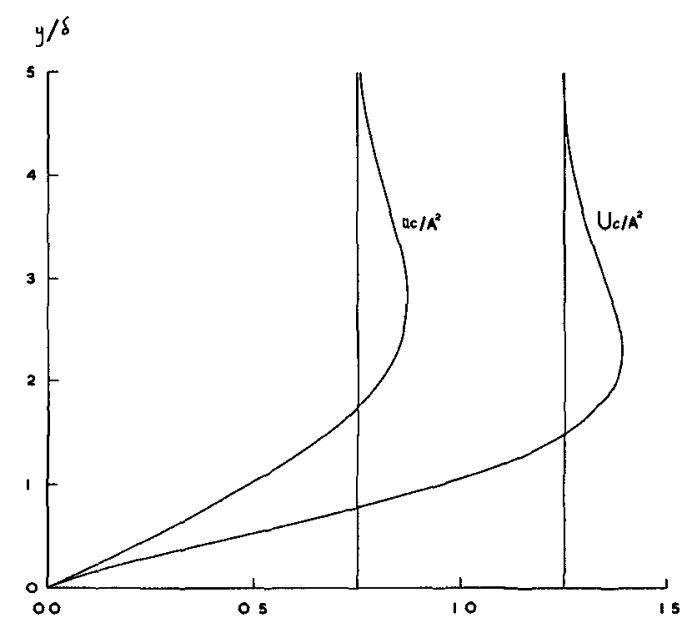

Fig. A5. The mean velocity $\bar{u}$ and the mass-transport velocity $U$ in the boundary-layer.

$\stackrel{U T}{\longrightarrow}$

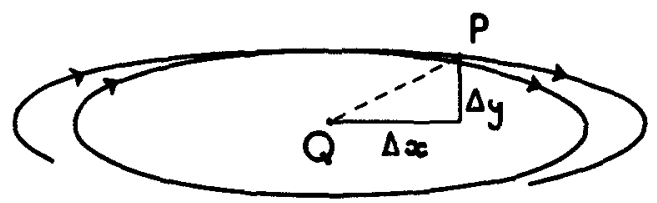

\section{$2 A / \%$}

Fig. A6. How the mass-transport velocity arises, when $\overline{\mathrm{u}}=0$. 


\section{COASTAL ENGINEERING}

Since $U$ vanishes on the bottom itself, this shows that $U$ must be positi very close to the bottom; here at least there is a forwards mass-transp velocity.

At other levels within the boundary-layer, however, the last two terms in eqn. (12) are not negliglble. For a progressive wave we have

$$
\overline{\frac{\partial u}{\partial x} \int u d t}=-\frac{1}{c} \overline{\frac{\partial u}{\partial t} \int u d t}=\frac{1}{c} \overline{u^{2}}
$$

by eqn. (21) below, and so from (10)

When $u \rightarrow u_{\infty}$ we have

$$
U=\frac{1}{c}\left(\overline{u_{\infty} u}+\frac{3}{2} \overline{u^{2}}\right) \text {. }
$$

$$
U \rightarrow \frac{5}{2 c} \overline{u_{\infty}^{2}}
$$

These remarkably simple formulae are independent of the absolute value of the viscosity and even (as will be shown) indenendent of the form of the distribution of viscosity within the layer. However, in the specia. case when the viscosity is constant and the motion sinusoidal we have or substitution from (3)

$$
U=\frac{A^{2}}{4 c}\left(5-8 e^{-y / 6} \text { as } y / 8+3 e^{-2 y / 6}\right) \text {. }
$$

This distribution is shown by the second curve in Fig. A5. $U$ is always positive and has a maximum value

$$
U=1.376 \ldots A^{2} / c
$$

As $y / 6$ tends to infinity

$$
U \rightarrow 1.25 \quad A^{2} / \mathrm{c}
$$

compared with the limiting value

$$
\bar{u} \rightarrow 0.75 A^{2} / c
$$

for the mean velocity.

(4) Discussion We have remarked that the formulae (14) and (15) are independent of the distribution of viscosity within the layer, provided that the flow is laminar. Now for turbulent but steady boundary-layers it has been shown that the flow may be quite well aporoximated by the laminar velocity profile, provided that in the outer part of the layer the ordinary viscosity is replaced by a uniform coefficient of eddy viscosity $(6)$. Now if the eddy viscosity fluctuates according to the instantaneous velocity gradient, then an oscillatory boundary-layer will not be strictly comparable with a steady boundary-layer. If on the othe hand we assume that the eddy-viscosity of a small element of fluid does not fluctuate appreciably throughout a wave period it is possible to replace the ordinary kinematic viscosity $y$ by a coefficient which is constant for a particle, though varying with the mean distance of the 


\section{IN A CLOSED CHANNEL}

particle from the bottom. Oar result then indicates that the equation

$$
U_{\infty}=\frac{5}{2 c} \overline{u_{\infty}^{2}}
$$

for the velocity just outside the boundary-layer is valid also when the flow is turbulent.

Moreover this expression is valid even when the motion, though periodic is not strictly harmonic, as will happen with long waves in shallow water when the form of a solitary wave is aporoached. Suppose that the velocity near the bottom, instead of being simply harmonic is given by an expression of the form

$$
\begin{aligned}
u_{\infty}=A_{1} \cos (\sigma t-k x) & +A_{2} \cos 2(\sigma t-k x)+\cdots \\
& +B_{2} \sin 2(\sigma t-k x)+\cdots
\end{aligned}
$$

in which the coefficients may be deduced theoretically or found from observation by Fourier analysis. Then the above expression gives

$$
U_{\infty}=\frac{5}{4 c}\left(A_{1}^{2}+A_{2}^{2}+\cdots+B_{2}^{2}+B_{3}^{2}+\cdots\right) .
$$

Although the mass-transport velocity just outside the layer has been shown to be independent of $y$, eqn. (2) shows that the distribution of the velocity within the layer is certainly dependent on the form of the viscosity. For this reason the expression (17) for the maximum velocity within the layer may not be valid for a turbulent layer.

However, what is observed in practical experiments is less likely to be the maximum velocity than the velocity just outside the layer, where the velocity gradients are less steep - especially if the boundary layer is thus and observations are made with streaks of dye. For, a slender tongue of dye is less easy to observe than a diffused cloud moving forwards with a relatively uniform velocity. It is fortunate that the latter velocity appears to be more easily predictable.

(5) Proof of eqn. (10) Finally shall prove our statement that eqn. (10) is true independently of the viscosity. Although the argument can at some length be translated into physical terms, it is simpler at this stage to give an analytical proof.

We start from egn. (2) and (9), which are both valid even when the viscosity is a function of time and position. From (2) we have by integration with respect to $t$,

and therefore

$$
u=u_{\infty}+\int \frac{\partial}{\partial y}\left(\nu \frac{\partial u}{\partial y}\right) d t
$$

$$
\overline{u v}-(\overline{u v})_{\infty}=\overline{u_{\infty} v}+\overline{\int \frac{\partial}{\partial y}\left(\nu \frac{\partial u}{\partial y}\right) d t \cdot v}-(\overline{u v})_{\infty} .
$$




\section{COASTAL ENGINEERING}

Now from the equation of continuity

and so

$$
\frac{\partial v}{\partial y}=-\frac{\partial u}{\partial x}=\frac{1}{c} \frac{\partial u}{\partial t}
$$

$$
\overline{u_{\infty} v}-(\overline{u v})_{\infty}=\int_{\infty}^{y} \overline{u_{\infty} \frac{\partial v}{\partial y}} d y=\frac{1}{c} \int_{\infty}^{y} \overline{u_{\infty} \frac{\partial u}{\partial t}} d y
$$

where the limit $\infty$ denotes a value of $y$ large compared with $\delta$ but small compared with the wavelencth or total depth. On substituting for du/dt from eqn. (2) we have

$$
\overline{u_{\infty} v}-(\overline{u v})_{\infty}=\frac{1}{c} \int_{\infty}^{y} \overline{u_{\infty}\left[\frac{\partial u_{\infty}}{\partial t}+\frac{\partial}{\partial y}\left(\nu \frac{\partial u}{\partial y}\right)\right]} d y=\frac{1}{c} \overline{u_{\infty} 2}
$$

since $u_{\infty} . \partial u_{\infty} / \partial t$
two periodic identically zero. Further, if $f$ and $g$ denote any

$$
\overline{f \frac{\partial g}{\partial t}}+\overline{\frac{\partial f}{\partial f}}=\overline{\frac{\partial}{\partial t}(f g)}=\frac{1}{T}(f g)_{t=0}^{t=T}=0
$$

and so in any averaged product of this type the operator $\partial / \partial t$ may be transferred from one member to the other, provided the sign is reversed at the same time. Thus for example

$$
\overline{\int \frac{\partial}{\partial y}\left(\nu \frac{\partial u}{\partial y}\right) d t \cdot v}=-\overline{\frac{\partial}{\partial y}\left(\nu \frac{\partial u}{\partial y}\right) \int v d t} \text {. }
$$

On substituting these results in eqn. (9) we obtain

$$
\overline{\nu \frac{\partial u}{\partial y}}=\overline{\frac{1}{c} u_{\infty} \nu \frac{\partial u}{\partial y}}-\overline{\frac{\partial}{\partial y}\left(\nu \frac{\partial u}{\partial y}\right) \int v d t .}
$$

We assume that the viscosity is constant following a particle but is a function $N(Y)$ of the mean height $Y$ of the particle above the bottom, then at any fixed point $(x, y)$ the viscosity will be a slightly varying function of the time. To the first approximation

$$
\nu(x, y, t)=N(Y)-\frac{d N}{d Y} \Delta y=N-\frac{d N}{d Y} \int v d r .
$$

substituting in (22) and neglecting third-order terms we have

$$
\overline{\left(N-\frac{d N}{d Y} \int v d t\right) \frac{\partial u}{\partial y}}=\frac{1}{c} \overline{N u_{\infty} \frac{\partial u}{\partial y}}-\overline{\left(N \frac{\partial^{2} u}{\partial y^{2}}+\frac{d N}{d r} \frac{\partial u}{\partial y}\right) \int v d t}
$$

The terms involving $d N / d Y$ cancel, and on dividing through by $N$, which is a function of $\gamma$ only, we obtain

$$
\frac{\partial \bar{u}}{\partial y}=\frac{1}{c} \overline{u_{\infty} \frac{\partial u}{\partial y}}-\overline{\frac{\partial^{2} u}{\partial y^{2}} \int v d t} .
$$

This relation is entirely free from $N$. The last term can be witten a:

$$
-\frac{\partial}{\partial y}\left(\overline{\frac{\partial u}{\partial y} \int v d r}\right)+\overline{\frac{\partial u}{\partial y} \int \frac{\partial v}{\partial y} d t} \text {, }
$$




\section{AN EXPERIMENTAL INVESTIGATION OF DRIFT PROFILES}

IN A CLOSED CHANNEL

and since

$$
\overline{\frac{\partial u}{\partial y} \int \frac{\partial u}{\partial y} d t}=\overline{\frac{\partial u}{\partial y} \int \frac{1}{c} \frac{\partial u}{\partial t} d t}=\frac{1}{\frac{\partial u}{\partial y} u}=\frac{1}{2 c} \frac{\partial}{\partial y} \overline{u^{2}}
$$

we have

$$
\frac{\partial \bar{u}}{\partial y}=\frac{1}{c} \overline{u_{\infty} \frac{\partial u}{\partial y}}+\frac{1}{2 c} \frac{\partial}{\partial y} \overline{u^{2}}-\frac{\partial}{\partial y}\left(\overline{\frac{\partial u}{\partial y} \int v d t}\right) .
$$

On integrating from $y=0$, where $u$ and $v$ vanish, we find eqn. (10)

Since the relation (12) between $U$ and $\bar{u}$ is purely kinematical, it follows that eqn. (14) and (15) also are independent of $N$.

\section{ADDITIONAL REFERENCES}

(5) Lamb, H. Hydrodynamics, 6th ed. Cambridge U. Press, 1932.

(6) Clauser, F. H. The turbulent boundary layer. Advances in Applied Mechanics, Vol. 4, pp.1-51, New York, Academic Press, 1956. 